their high expression of MHC class II (HLA-DR) and co-stimulatory (CD80 and CD86) molecules suggests an important antigen-presentation function of $A B C$, which together with their unique FcRL family expression pattern, warrants further functional characterisation.

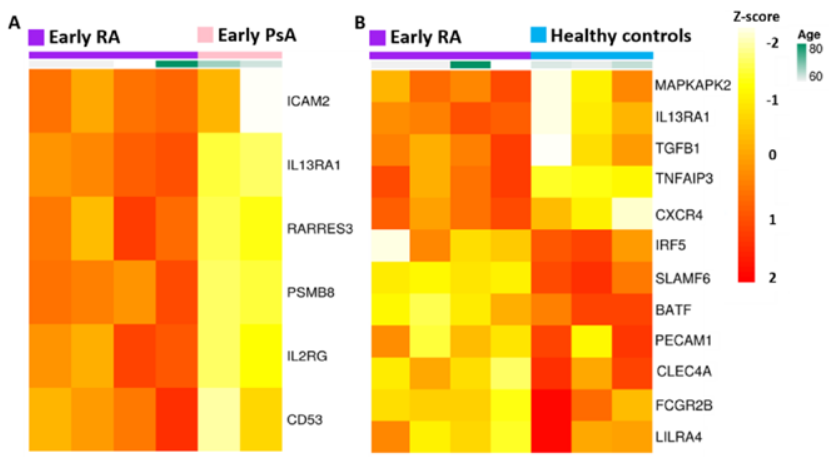

Figure 1. Differential gene expression in $A B C$ s from early $R A$ patients compared to early PsA patients (A) and healthy controls (B). A NanoString nCounter Technologies chip was used to assess gene expression. Raw counts were normalised to the housekeeping genes. Sample quality was then assessed using the arrayQualityMetrics package. Gene expression profiles between different donor groups was assessed using the DESeq2 R package. In both hierarchical clustering heatmaps gene expression intensities were log2 transformed and their z-scores are displayed as colours ranging from yellow (low expression) to red (high expression) as shown in the key.

Disclosure of Interests: Gemma Vidal-Pedrola: None declared, Najib Naamane: None declared, Dagmar Scheel-Toellner: None declared, Arthur Pratt Grant/research support from: GSK, Pfizer and Janssen, Andrew Mellor: None declared, John D Isaacs Speakers bureau: Abbvie, Gilead, Roche, UC, Consultant of: Abbvie, Gilead, Roche, UC, Grant/research support from: Pfizer, GSK and Janssen, Amy E. Anderson Grant/research support from: Pfizer, GSK and Janssen

DOI: 10.1136/annrheumdis-2021-eular.2010

\section{AB0022 AUTOREACTIVE B CELLS IN RHEUMATOID ARTHRITIS DISPLAY AN ACTIVATED PHENOTYPE OF RECENT ANTIGEN EXPOSURE}

N. Blomberg ${ }^{1}$, H. Kristyanto ${ }^{1}$, T. Huizinga ${ }^{1}$, R. Toes ${ }^{1}$, H. U. Scherer ${ }^{1} .{ }^{1}$ Leiden University Medical Center (LUMC), Rheumatology, Leiden, Netherlands

Background: Rheumatoid arthritis, in particular ACPA+ RA, is characterized by frequent disease flares and poor chances to achieve DMARD-free sustained remission. Recently, we have shown that ACPA-expressing memory B cells (MBC) remain in a persistently activated state throughout disease, even in patients in DMARD-induced clinical remission.(1) The reasons why the ACPA $B$ cell response is continuously activated are unknown, as well as why the response does not revert to a resting, 'quiescent' state. We hypothesized that continuous antigen exposure in germinal centres drives ACPA B cell activation, leading to a 'recent germinal centre emigrant' phenotype of these cells in the circulation

Objectives: To understand whether the activated phenotype of ACPA-expressing B cells could be induced by recent antigen exposure, to thereby discern the processes of immune activation that remain active in patients even in clinical remission and to argue whether these processes could be targets for therapeutic intervention.

Methods: ACPA-expressing B cells were identified in peripheral blood of RA patients by flow cytometry during different stages of disease and characterized by a panel of activation- and germinal centre related markers (CD80, CD86, CD32, CD95, Ki-67). In addition, three healthy donors received a TT booster vaccination. TT-specific MBC were identified in blood at different timepoints (before vaccination and up to 22 weeks after vaccination) and analysed phenotypically over time.

Results: The majority of ACPA-expressing B cells strongly expressed CD95 and the co-stimulatory marker $\mathrm{CD} 80$. A part was also positive for the proliferation marker Ki-67 (on average 30\%), and most cells downregulated the inhibitory marker CD32. TT-specific MBC adopted a comparable phenotype after booster vaccination, but most markers returned to the pre-vaccination expression level gradually over time. These effects were antigen-dependent because the phenotype of TT-negative $B$ cells remained unchanged. The phenotypic composition of the proliferating ACPA-positive B cell pool most closely corresponded to a stimulation history of 1-2 weeks after antigen exposure. Notably, none of the Ki-67 negative ACPA-specific MBC showed phenotypic quiescence, indicating either a short life-time (in circulation) after antigen encounter or persistent additional factors of activation.
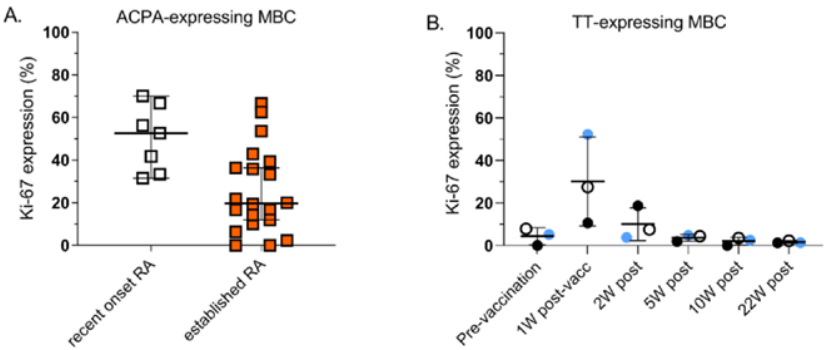

Figure 1. Ki-67 expression on ACPA-specific MBC in RA (A) and on TT-specific MBC in 3 healthy donors before and after booster vaccination $(B)$.

Conclusion: ACPA-expressing MBC phenotypically resemble TT-specific MBC after recent (1-2 weeks) booster vaccination, reflecting the phenotype of recent germinal centre emigrants, and remain activated, whereas TT-specific MBC lose this marker profile over time. These observations suggest that ACPA-expressing MBC either home to tissue or survive shortly in the circulation, or that additional factors drive or program these cells to persistent activation. Transcriptomic profiling and analysis of the homing marker profile may help to answer these questions. Furthermore, it will be important to understand the association of persistent activation of ACPA-expressing B cells in clinical remission and the risk for disease flares upon treatment discontinuation.

\section{REFERENCES:}

[1] Kristyanto H, Blomberg NJ, Slot LM, van der Voort ElH, Kerkman PF, Bakker A, et al. Persistently activated, proliferative memory autoreactive B cells promote inflammation in rheumatoid arthritis. Sci Transl Med. 2020;12(570). Disclosure of Interests: Nienke Blomberg: None declared, Hendy Kristyanto: None declared, Thomas Huizinga Grant/research support from: Gilead, Rene Toes: None declared, Hans Ulrich Scherer Grant/research support from: Pfizer, Lilly, Sanofi, BMS DOI: 10.1136/annrheumdis-2021-eular.2050 \section{DENDRITIC CELLS AS A PROMINENT MARKERS OF AUTOIMMUNE DISEASES}

M. Korolev ${ }^{1}$, Y. Kurochkina ${ }^{1}$, N. Banshhikova ${ }^{1}$, V. Omelchenko ${ }^{1}$, A. Akimova ${ }^{1}$, E. Letyagina ${ }^{1}$, O. Poveschenko ${ }^{2} .{ }^{1}$ Research Institute of Clinical and Experimental Lymphology - Branch of the Institute of Cytology and Genetics, Siberian Branch of the Russian Academy of Science, Rheumatology, Novosibirsk, Russian Federation; ${ }^{2}$ Research Institute of Clinical and Experimental Lymphology Branch of the Institute of Cytology and Genetics, Siberian Branch of the Russian Academy of Science, Cells Technologies, Novosibirsk, Russian Federation

Background: Dendritic cells (DCs) are known to contribute to the pathogenesis of different autoimmune diseases. It is clear nowadays the role of DCs in rheumatoid arthritis (RA) but not well investigated in Axial spondylitis (AxSpA). DCs are a heterogeneous population and can be divided into groups: myeloid (mDCs) and plasmacytoid (pDCs). DCs can induce both immune response and tolerance. Objectives: To investigate the subpopulations of peripheral blood myeloid and plasmacytoid DCs in patients with early stage of RA (duration of illness up to 12 months) and AS.

Methods: The study include sixty five patients with early forms of diseases including 55 patients with RA and 10 patients with AxSpA. Diagnosis RA was established according ACR/EULAR criteria (2010). Diagnosis AxSpA was established according ASAS criteria. All patients received conventional synthetic DMARDs. Thirty patients with osteoarthritis $(\mathrm{OA})$ used as a control group. Analysis of the content of the B-lymphocytes, myeloid and plasmacytoid DCs was carried out by flow cytometry. B-lymphocytes, subtypes of peripheral blood DCs were characterized by the following phenotypes: myeloid DCs (CD3-CD14-CD19-HLA-DR + CD11C + CD123-) plasmacytoid DCs (CD3-CD14-CD19-HLA-DR + CD11C-CD123 +), B-lymphocytes $(C D 19+)$. Analyses were performed before treatment and after 3 and 6 months. Results: Patients with early RA are characterized by significant evaluation of the population of myeloid DCs in comparison of patients with osteoarthritis $(25.3 \%$ vs $21.5, p=0.005)$. Furthermore, the difference was found in the number of cells with the phenotype B-lymphocytes: $5.7 \%$ vs $3.1 \%, p=0.0007$ ). No significant differences were observed in the number of plasmocytoid DCs. After 3 and 6 month of observation we detected reducing amount of myeloid DCs $26.7 \%$ vs $20.1 \%$ vs $16.4 \%$ respectively. Disease activity according to DAS28 droped to low $(4.32$ to3.06, $\mathrm{p}=0.03$ ). Patients with AxSpA are characterized a lower mDCs levels in compared with RA (19.3\% vs 26.7, $\mathrm{p}=0.07)$. After 6 month of investigation we detected decreasing $\mathrm{mDCs}(19.3 \%$ to $14.2 \%, \mathrm{p}=0.05)$. The percent of $\mathrm{pDCs}$ were constant and did not differ from the level of healthy donors.

Conclusion: The data obtained indicate that early form of rheumatic diseases namely rheumatoid arthritis and axial spondylitis have the common features such as the dominance of $\mathrm{mDCs}$ and their decreasing in reduction of activity of disease. Disclosure of Interests: None declared

DOI: 10.1136/annrheumdis-2021-eular.2293 